\title{
Automated defect detection based on time and frequency domain analysis using Eddy Current Pulsed Thermography in EB and TIG welding inspections.
}

\author{
by R. Hidalgo-Gato, E. Gorostegui-Colinas, P. López de Uralde, A. Muniategui, B. Urtasun, I. Silanes \\ LORTEK Technological Centre, Basque Research and Technology Alliance (BRTA), Arranomendia kalea, 4A, \\ Ordizia, 20240, Spain, rhidalgo@lortek.es
}

\begin{abstract}
On this work an active thermography has been applied to Inconel 718 EBW and TIG samples, continuing previous research on this field [1, 2]. In particular, the objective of this work is to improve and automate the processing part of the automatic defect detection procedure to enhance the results of the detection algorithm, which is the one applied in the last step. The proposed method is based on a time and frequency domain analysis (TFDA), in which, the resulting thermograms contain the most relevant thermal information regarding to the defect thermal evolution. To assess the suitability on each resulting thermogram (including the fused images), an accuracy evaluation based on Tanimoto criterion has been employed. This evaluation leads to an optimate processing procedure that ensures a detection with less false positives or negatives.
\end{abstract}

\section{Introduction}

The inspection of welded components is a key step of the manufacturing process in the aerospace industry to ensure high quality products. Fusion welding is one of the most frequently used methods for joining metal parts in the aerospace industry. In this work, the metal parts under study (Inconel 718) are welded using two techniques: Tungsten Inert Gas Welding (TIG) and Electron Beam Welding (EBW) [3]. In the TIG process a non-consumable tungsten electrode, shielded by inert gas, is used to strike an electric arc with the base metal. The heat generated by the electric arc is used to melt and join the base metal [4]. EBW process occurs by joining metallic materials by melting the area of a welded joint in high vacuum, using a beam of high velocity electrons [5].

The presence of defects in the welded components is critical and may drive to integrity failures with catastrophic consequences. Without any visible and previous warning, surface and subsurface cracks in metals can appear and extend, leading to a complete fracture of the component. For this reason, a robust inspection method is sought in order to assure its complete safety. The most common NDT inspection methods used in aerospace industry are: Visual inspection (VT), Dye penetrant testing (PT), Magnetic particle testing (MT), Ultrasonic testing (UT) and Radiography (RT). Conventional NDT methods are usually based on manual techniques that are really time-consuming and inspectors must be highly qualified. Furthermore, some of these procedures have requirements regarding waste management due to the hazardous products used during the inspection process [6]. Finally, the biggest limitation can be found in the automatization, something invaluable that dye penetrant testing or magnetic particles will never allow. All those restrictions highlight the importance of finding an alternative NDT inspection technique, clean and easy automatized.

Taking into account the human and environmental impact mentioned above and the key statement for the detection of surface defects, this work proposes an alternative NDT inspection technique based on active thermography. Thermal inspection based on IR radiance provides fast, contactless and safe measurements. It also offers the possibility to automate both the inspection and detection process. Furthermore, several excitations sources can be employed depending on the material to be inspected: electromagnetic, mechanical or optical [7]. As for the excitation mode, it can be based on a pulse or lock-in thermography. In this case, the previous work on this type of welding has led us to choose Eddy Current Thermography (or Inductive Thermography) [7] [1] [2].

Eddy current thermography technique is based on the thermal effect that arises due to the eddy currents induced in the material. The generated currents are converted to heat according to Joule's law. The material's voids or discontinuities can be detected analysing the surface thermal contrast generated by the distortions in the current flow distribution. The Eddy current pulsed thermography (ECPT) method provides an excellent surface and subsurface crack detection sensitivity in metals due to their electrical and thermal conductivity and high permeability using a very short electromagnetic pulse (less than $500 \mu s$ ) [8].

\section{Processing methodology}

In this article, a previous automated defect detection algorithm [1] [2] has been improved for the detection of cracks on Inconel 718 EB and TIG welded components. This enhancement also affects the step prior to the detection: the processing. Thus, an hybrid method is proposed (see flow chart in Fig. 1), it is formed by three parts: 
- $\quad$ Pre-processing: to reduce signal degradation and noise (based on bad pixel correction and fixed-pattern noise correction [FPNr]), which will prepare the thermal signal for the next step (processing).

- $\quad$ Processing: based on time and frequency domain analysis (TFDA) and image fusion.

- $\quad$ Detection: based on Polar Discrete Fourier Transformation (pDFT).

The processing part combines the time and frequency domain analysis to obtain two thermograms on each domain with complementary thermal information regarding the defects on the material. On the resulting thermograms the butterfly patterns induced by defects are enhanced. The detection algorithm (pDFT) is used as a reference to compare the suitability of all the processing procedure. This comparison analysis is based on the Tanimoto Criterion (TC) using the coordinates generated by the pDFT for the defect detection [9]. In order to automate the process, the defects zones in all samples are pre-defined (10x10 pixels), thus, the TC can be applied automatically in all cases. Finally, an image fusion (based on Mertens exposure fusion algorithm) is performed on the TFDA thermograms to analyse the detection accuracy of the output thermograms, seeking to obtain in a single thermogram the best pDFT input to ensure the detection of all the defects [10] [11].

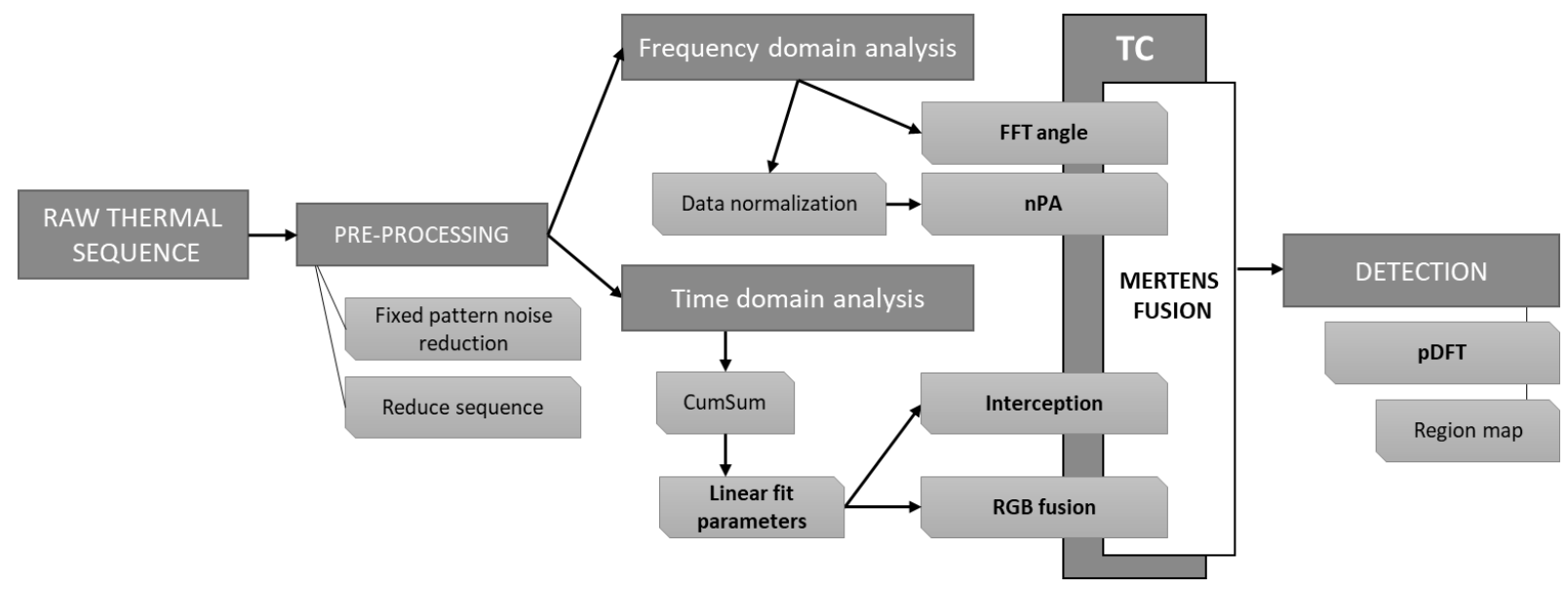

Fig. 1. Proposed hybrid method flow chart.

\subsection{Frequency domain analysis}

The frequency domain thermal sequence analysis is based on Fast Fourier Transform phase calculation (FFTa) and the pre-normalized peak amplitude values reconstruction (nPA).

\section{Fast Fourier Transform angle phase (FFTa)}

Fast Fourier Transform enables to change from time domain to frequency domain, analysing the time evolution of the image sequence captured by pulsed infrared thermography applying the discrete one-dimensional Fourier transform pixel-by-pixel [12] [13]. The FFT is a collection of algorithms for fast computation of the Discrete Fourier Transform (DFT) and is based on factorizations to reduce the transforms complexity. The FFT algorithm implemented in this work has been the Cooley-Tukey algorithm [14] [15]. The one-dimensional function decomposes the surface thermal signal evolution in two components: amplitude (modulus) and phase (phase delay), both symmetrical about the frequency $f=0 \mathrm{~Hz}(n=\mathrm{N} / 2)$. The discrete FT (DFT) and its angle phase can be written as in equations 1-2. The resulting thermogram (phasegram) contains the first non-zero phase frequencies.

$$
\begin{aligned}
& F_{n}=\Delta t \sum_{k=0}^{N-1} T(k \Delta t) \exp ^{(-\mathrm{j} 2 \pi n k / N)}=R e_{n}+I m_{n} \\
& \emptyset_{n}=\tan ^{-1}\left(\frac{I m_{n}}{R e_{n}}\right)
\end{aligned}
$$

\section{Pre-normalized peak amplitude values reconstruction (nPA)}

The scaled peak amplitude value calculation uses the thermal signal amplitude component obtained by FFT. In this case a data normalization was applied before the FFT calculation (to enhance the thermal contrast). Once the data is normalized, the amplitude in the frequency domain of the thermal evolution is evaluated to construct a single thermogram containing the peak amplitude value. 
This pre-normalization, scales the thermal sequence pixel-by-pixel to a unit norm as follows:

$$
\|v\|_{p}=\left[\sum_{k=1}^{N}\left|v_{k}\right|^{p}\right]^{1 / p}
$$

Where $\mathrm{N}$ is the number of thermograms and $\mathrm{p}=2$ (L2 norm). The normalized data (the thermal signal evolution in time) is processed by FFT applied pixel-by-pixel, resulting in an FFT peak amplitude thermogram which contains the peak amplitude values. The phase is not affected by the data normalization but the amplitude is scaled to peaks with the highest ratios due to the correction over the spectral range (normalized FFT spectrum).

\subsection{Time domain analysis}

The time domain thermal sequence analysis is based on a linear regression evaluation of the material's surface thermal response evolution in time. The evaluated thermal vector, comprises from the first valid thermogram (after FPNr) to the halfway point of the cooling part (reduce sequence). Once the thermal sequence is pre-processed an accumulative sum algorithm is applied to adapt the signal and avoid thermal peak curves. The following step involves a linear regression evaluation (LRE) for each pixel in the time domain. Three thermograms are reconstructed (curve slope, origin, and $R^{2}$ ) from the resulting linear regression parameters and the output image the RGB fusion of these three regression parameters (see Fig. 3). Also, one more resulting thermogram is added to the LRE, based on the first intersection time instant between the original thermal curve and its linear artificial reconstruction (LRE_int). All plotted images use an auto-contrast algorithm based on image percentile calculation and scale normalization.

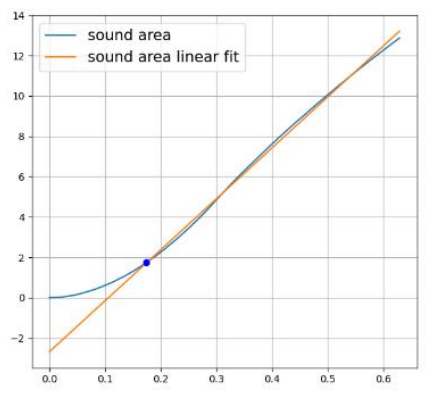

(a)

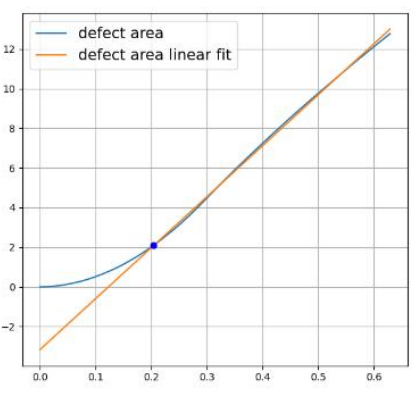

(b)

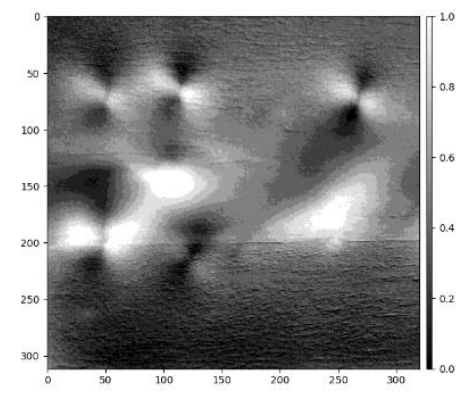

(c)

Fig. 2. LRE intersection time instant calculation for a sound area (a) defected area (b) and the thermogram reconstruction (sample Sa1).
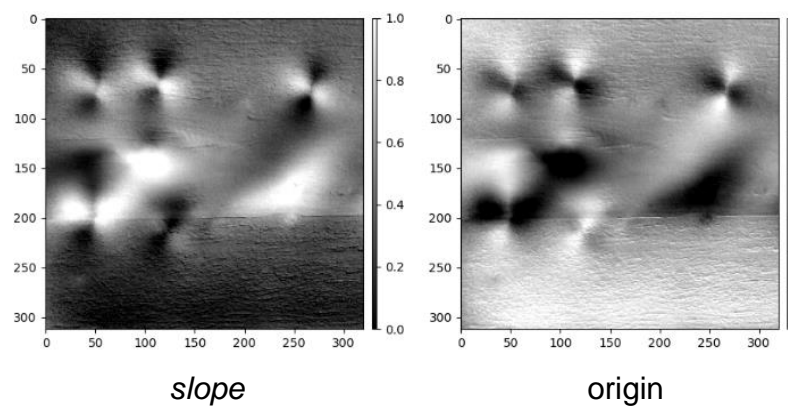

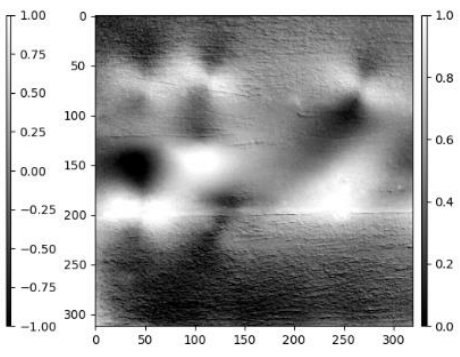

$\mathrm{R}^{2}$

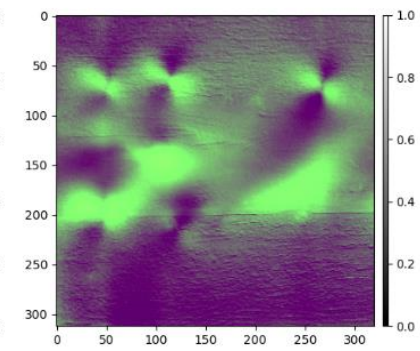

RGB

Fig. 3. RGB fusion using as example Sa1.

\section{Experimental setup and data acquisition}

The experimental tests were performed using a FLIR X6540sc MWIR camera with a full resolution of $640 \times 512$ pixels (at $126 \mathrm{~Hz}$ ). The eddy current pulsed thermography (ECPT) inspection was conducted using a $3 \mathrm{~kW}$ induction module from Edevis $\mathrm{GmbH}$ and a square inductor manufactured at Lortek (see Fig. 4) with an excitation frequency of $30 \mathrm{kHz}$ and a pulse length of $300 \mathrm{~ms}$ with a camera frame rate of $300 \mathrm{~Hz}$ (using subwindowing). The inductor has a square ferrite core with an internal window of $34 \times 34 \mathrm{~mm}$ and an external area of $50 \times 50 \mathrm{~mm}$. The coil was manufactured using Litz wires to improve insulation and reduce the thermal reflexion over the measured area due to coil cable heat loss. Litz wires are made of individually enamel-insulated strands of copper and each strand in a bundle is usually coated with an insulating material to prevent its electrically contact with the strands around it [2]. 


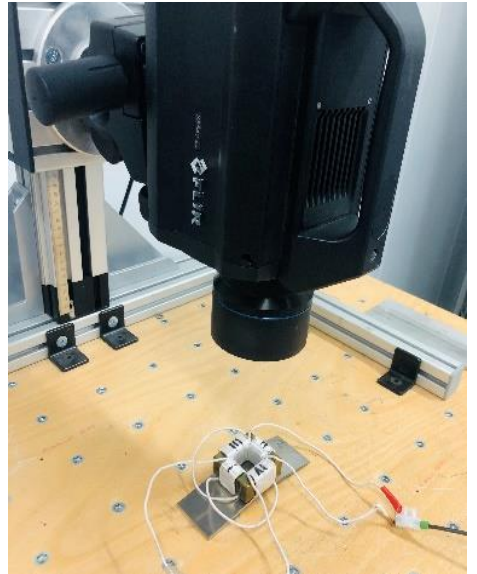

(a)
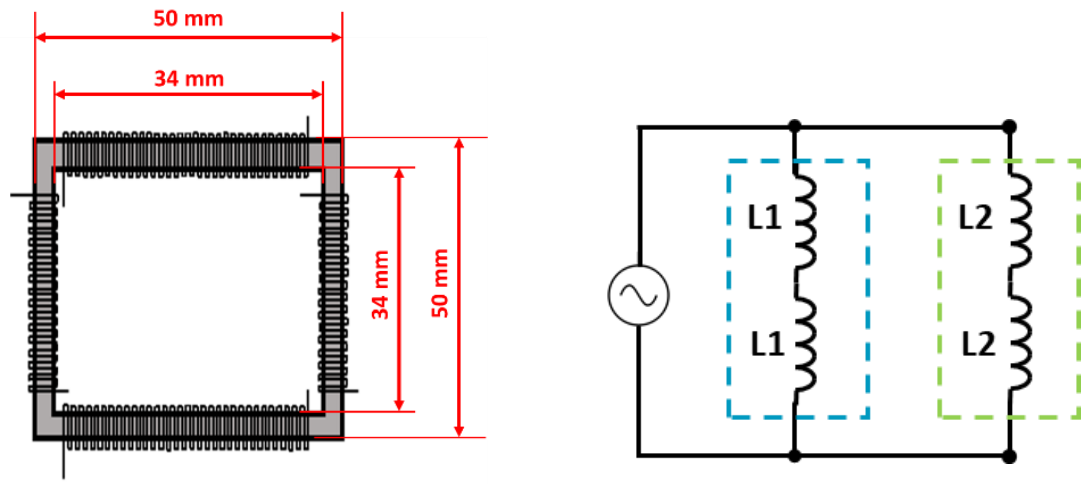

(b)

Fig. 4. Experimental setup (a) and inductor configuration (b).

In this study, the experiment was tested on four samples (see Fig. 5): Samples Sa1 is an EB welded sample, while sample Sa2, Sa3 and Sa4 have been manufactured in the Varestraint machine at Lortek. All four samples are only different in geometry and defects.

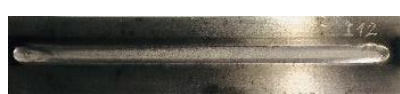

Sa1
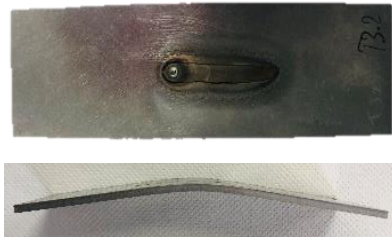

Sa3

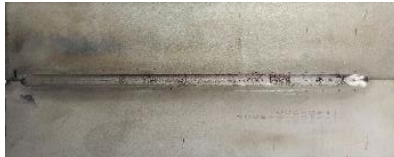

Sa2
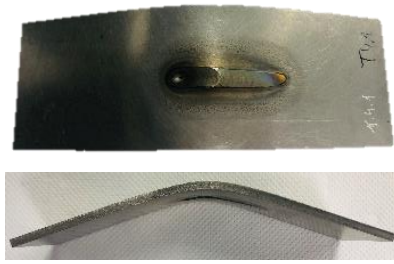

Sa4

Fig. 5. Tested samples.

In the next section, the study results are presented, following the proposed method in which the four resulting thermograms from the time and frequency analysis (TFDA) are evaluated.

\section{Results and discussion}

In order to evaluate the suitability of the TFDA resulting thermograms, a comparative analysis has been performed using the Tanimoto criterion (TC). For each sample, the defective zones and the total number of defects have been predefined to help to automate the TC calculation. The automated accuracy evaluation procedure uses the detection algorithm (pDFT) to obtain the defects coordinates. Noting that our main goal is to enhance the processing output to ensure the detection accuracy, the pDFT parameters employed have been the same for the entire procedure. Thus, if the detection accuracy is good, the processed thermogram is correct.

\subsection{Accuracy evaluation}

The TFDA thermograms are comprised by: FFTa (first non-zero phase frequencies), nPA (scaled peak amplitude value), LRE_int (time instant for the first regression intersection) and the RGB fusion of the LRE parameters. The Tanimoto criterion used in the accuracy evaluation has been defined as follows [9]:

$$
\mathrm{T}_{\mathrm{c}}=\frac{\mathrm{N}_{\mathrm{r} . \mathrm{d}}-\mathrm{N}_{\mathrm{m} . \mathrm{d}}}{\mathrm{N}_{\mathrm{r} . \mathrm{d}}+\mathrm{N}_{\mathrm{f} . \mathrm{d}}}
$$

where $N_{r . d}, N_{m . d}$ and $N_{f . d}$ are the numbers of true, missed and false defects detected by the pDFT algorithm. 

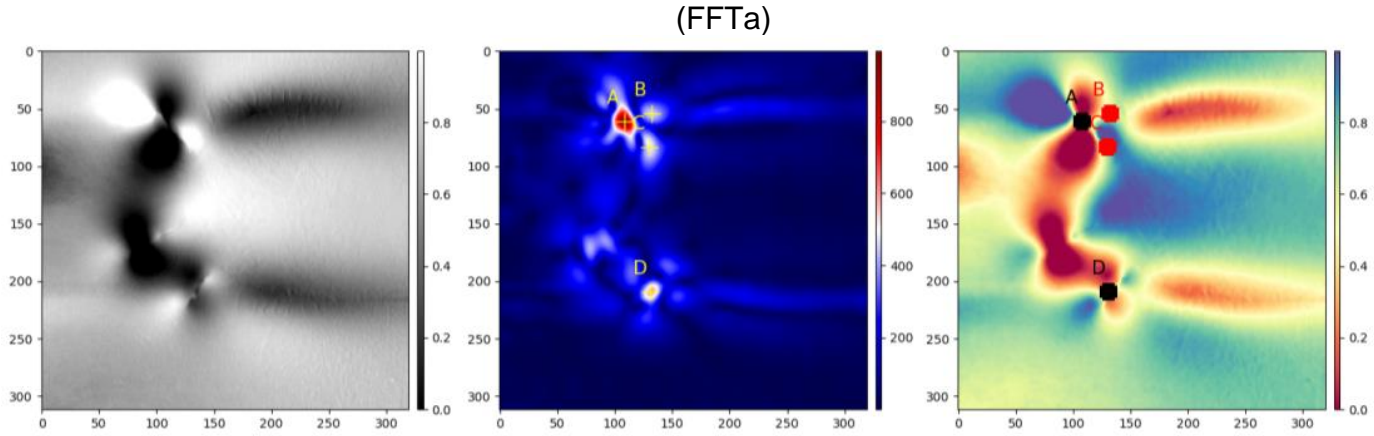

(nPA)

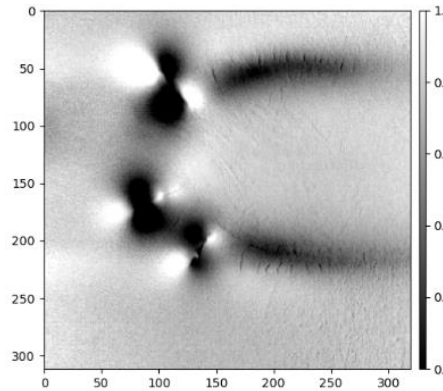

(a)

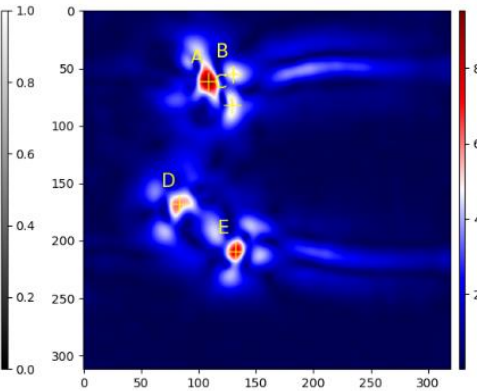

(b)

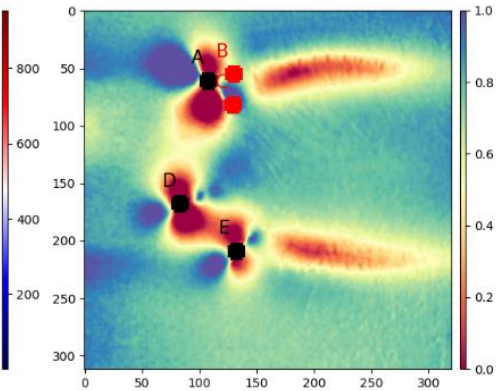

(c)

Fig. 6. Accuracy evaluation process example (Sa4). Thermogram obtained (a), pDFT regions map (b) and final plot based on TC (c).

As shown in Fig. 6, the accuracy evaluation based on TC uses the pDFT as reference to determine the defects coordinates. Using the pre-defined defective zones for each sample, the TC has been calculated and plotted (see Fig. 6c). The true defects have been plotted in black and false defects in red. Missed defects have not been plotted. As can be seen in Table 1 the first thermograms evaluated have been the ones obtained in the LRE procedure. The aim is to ensure that the RGB fusion performed over the reconstructed thermograms complements the defects signal variations identified on each LRE parameter.

Table 1. RGB analysis for all samples, based on TC.

\begin{tabular}{|c|c|c|c|c|c|}
\hline \multicolumn{7}{|c|}{ Sa1 } \\
\hline & $\mathbf{N}_{\mathbf{r d}}$ & $\mathbf{N f}_{\mathbf{d}}$ & $\mathbf{N}_{\text {md }}$ & $\mathbf{R}_{\mathbf{d t}}$ & $\mathbf{T C}[\%]$ \\
\hline Slope & 5 & 0 & 0 & 5 & 100 \\
\hline Origin & 5 & 0 & 0 & 5 & 100 \\
\hline $\mathrm{R}^{2}$ & 5 & 1 & 0 & 5 & 83.33 \\
\hline RGB & 5 & 0 & 0 & 5 & 100 \\
\hline
\end{tabular}

\begin{tabular}{|c|c|c|c|c|c|}
\hline \multicolumn{7}{|c|}{$\mathrm{Sa2}$} \\
\hline & $\mathbf{N}_{\mathbf{r d}}$ & $\mathbf{N f}_{\mathbf{d}}$ & $\mathbf{N}_{\mathbf{m d}}$ & $\mathbf{R}_{\mathbf{d t}}$ & $\mathbf{T C}[\%]$ \\
\hline Slope & 5 & 0 & 0 & 5 & 100 \\
\hline Origin & 5 & 0 & 0 & 5 & 100 \\
\hline $\mathrm{R}^{2}$ & 1 & 4 & 4 & 5 & -60 \\
\hline RGB & 5 & 0 & 0 & 5 & 100 \\
\hline
\end{tabular}

\begin{tabular}{|c|c|c|c|c|c|}
\hline \multicolumn{7}{|c|}{ Sa3 } \\
\hline & $\mathbf{N}_{\mathbf{r d}}$ & $\mathbf{N f}_{\mathbf{d}}$ & $\mathbf{N}_{\text {md }}$ & $\mathbf{R}_{\mathbf{d t}}$ & $\mathbf{T C}[\%]$ \\
\hline Slope & 3 & 1 & 0 & 3 & 75 \\
\hline Origin & 3 & 2 & 0 & 3 & 60 \\
\hline $\mathrm{R}^{2}$ & 2 & 3 & 1 & 3 & 20 \\
\hline RGB & 3 & 0 & 0 & 3 & 100 \\
\hline
\end{tabular}

\begin{tabular}{|c|c|c|c|c|c|}
\hline \multicolumn{7}{|c|}{ Sa4 } \\
\hline & $\mathbf{N}_{\text {rd }}$ & $\mathbf{N f}_{\mathbf{d}}$ & $\mathbf{N}_{\text {md }}$ & $\mathbf{R}_{\mathbf{d t}}$ & $\mathbf{T C}[\%]$ \\
\hline Slope & 3 & 2 & 0 & 3 & 60 \\
\hline Origin & 3 & 2 & 0 & 3 & 60 \\
\hline $\mathrm{R}^{2}$ & 2 & 2 & 1 & 3 & 25 \\
\hline RGB & 3 & 2 & 0 & 3 & 60 \\
\hline
\end{tabular}

The detection accuracy is higher in samples Sa1 and Sa2 and the statistical fit goodness R-squared value represents the lowest detection accuracy for all samples. However, the RGB thermogram is able to maintain or increase the detection quality. The detection quality of Samples Sa3 and Sa4 is limited due to the false defects caused by a longer butterfly thermal patterns generated on the materials surface. 
FFTa
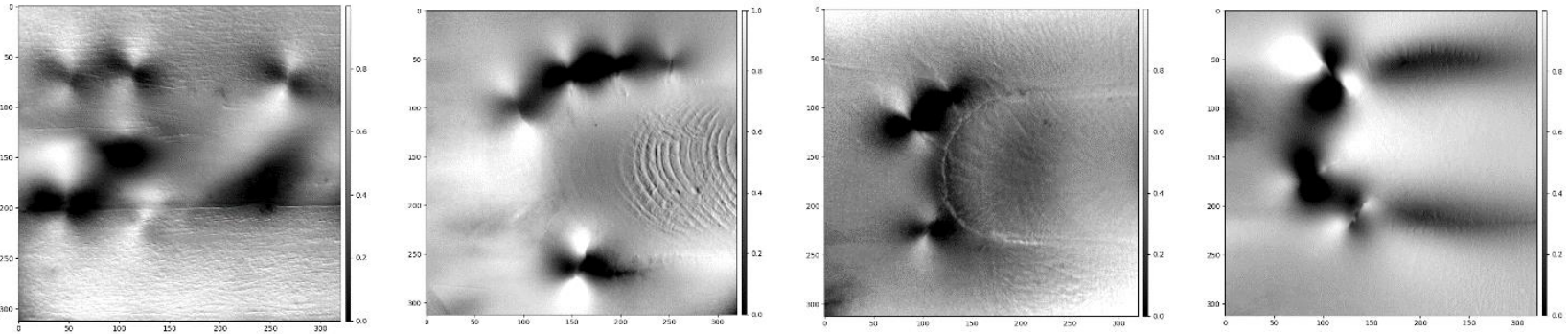

nPA
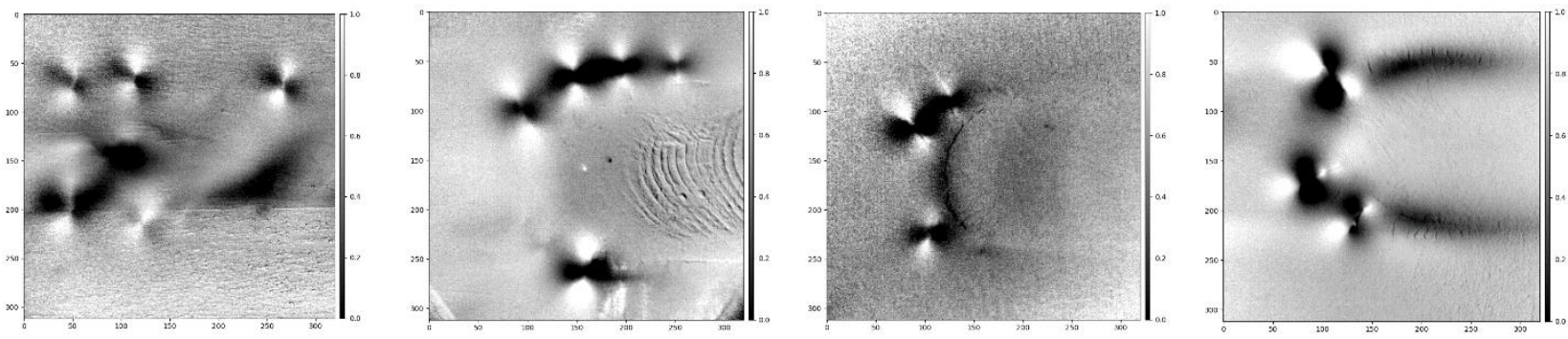

LRE_int
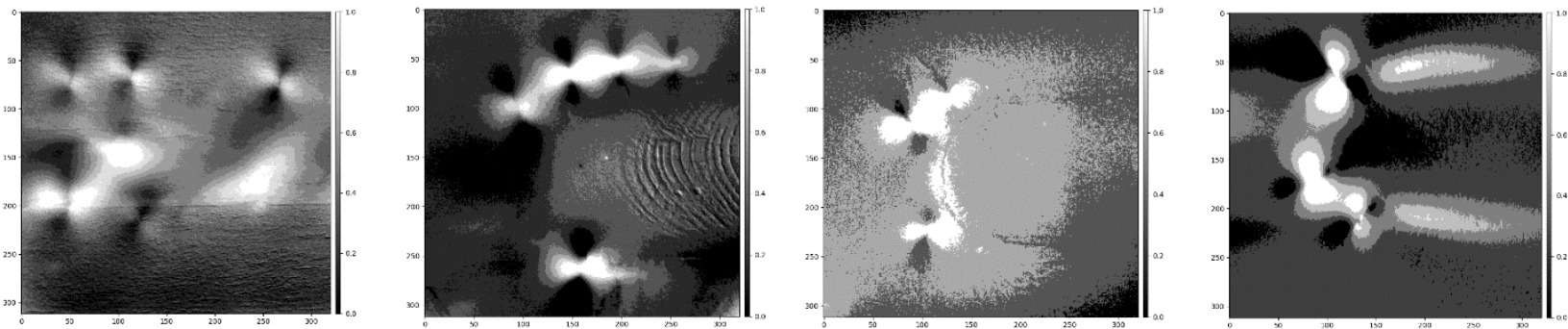

\section{LRE RGB}

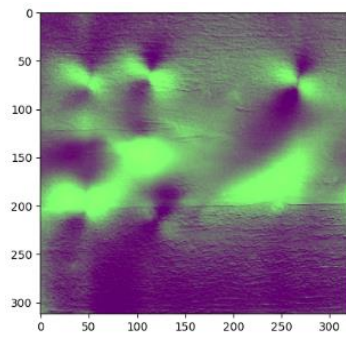

Sa1

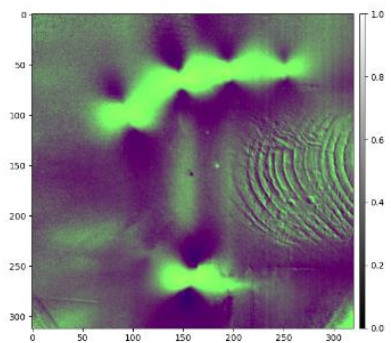

Sa2

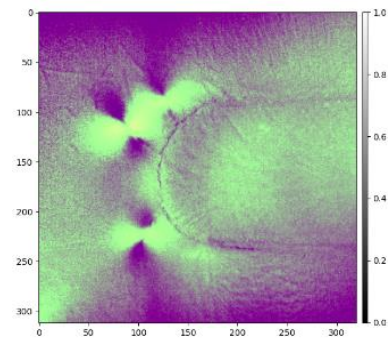

Sa3

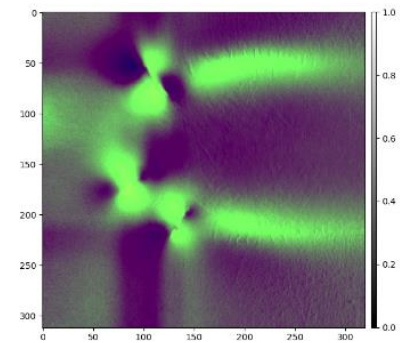

Sa4

Fig. 7. TFDA output thermograms for the selected samples.

Figure above shows all outputs thermograms after applying the proposed TFDA processing method. The optimized algorithms generate very good results identifying all defects contained on each sample. Likewise, the TC evaluation results show a high defect detectability, only limited in Sa4 where the sample geometry leads to the appearance of false positives and one defect is missing (false negative) in two of the four thermograms. Once again, the best results are obtained for samples Sa1 and Sa2 where the TC parameter reaches the highest values. In sample Sa3, all defects are detected with no missing defects. Detection is only limited by the appearance of some false positives. 
Taking into account the TC evaluation results, the TFDA thermograms with the highest accuracy are the nPA and the LRE_RGB. Even in sample Sa4 which has the lowest detection accuracy, all real defects are detected with both algorithms.

Table 2. Processing algorithms TC comparison.

\begin{tabular}{|c|c|c|c|c|c|c|c|c|c|c|c|}
\hline \multicolumn{6}{|c|}{ Sa1 } & \multicolumn{6}{|c|}{$\mathrm{Sa} 2$} \\
\hline & $N_{r d}$ & $\mathbf{N f}_{\mathrm{d}}$ & $N_{m d}$ & $\mathbf{R}_{\mathrm{dt}}$ & TC [\%] & & $N_{r d}$ & $\mathrm{Nf}_{\mathrm{d}}$ & $\mathrm{N}_{\mathrm{md}}$ & $\mathbf{R}_{\mathrm{dt}}$ & TC [\%] \\
\hline FFTa & 4 & 0 & 1 & 5 & 75 & FFTa & 5 & 0 & 0 & 5 & 100 \\
\hline nPA & 5 & 0 & 0 & 5 & 100 & $n P A$ & 5 & 0 & 0 & 5 & 100 \\
\hline LRE_int & 5 & 0 & 0 & 5 & 100 & LRE_int & 5 & 0 & 0 & 5 & 100 \\
\hline LRE_RGB & 5 & 0 & 0 & 5 & 100 & LRE_RGB & 5 & 0 & 0 & 5 & 100 \\
\hline
\end{tabular}

\begin{tabular}{|c|c|c|c|c|c|}
\hline \multicolumn{7}{|c|}{ Sa3 } \\
\hline & $\mathbf{N}_{\text {rd }}$ & $\mathbf{N f}_{\mathbf{d}}$ & $\mathbf{N}_{\text {md }}$ & $\mathbf{R}_{\mathbf{d t}}$ & TC [\%] \\
\hline FFTa & 3 & 1 & 0 & 3 & 75 \\
\hline nPA & 3 & 1 & 0 & 3 & 75 \\
\hline LRE_int & 3 & 2 & 0 & 3 & 60 \\
\hline LRE_RGB & 3 & 0 & 0 & 3 & 100 \\
\hline
\end{tabular}

\begin{tabular}{|c|c|c|c|c|c|}
\hline \multicolumn{7}{|c|}{ Sa4 } \\
\hline & $\mathbf{N}_{\text {rd }}$ & $\mathbf{N f}_{\mathbf{d}}$ & $\mathbf{N}_{\text {md }}$ & $\mathbf{R}_{\mathbf{d t}}$ & TC [\%] \\
\hline FFTa & 2 & 2 & 1 & 3 & 25 \\
\hline nPA & 3 & 2 & 0 & 3 & 60 \\
\hline LRE_int & 2 & 2 & 1 & 3 & 25 \\
\hline LRE_RGB & 3 & 2 & 0 & 3 & 60 \\
\hline
\end{tabular}

\subsection{Mertens fusion evaluation}

The Mertens fusion method is based on an exposure fusion algorithm developed to blend multiple exposures photographic images to enhance the capture scene dynamic range, also called high dynamic range imaging (HDR). Mertens blending has been defined as follows [10].

$$
L\{R\}_{i j}^{l}=\sum_{k=1}^{N} G\{\widehat{W}\}_{i, j, k}^{l} L\{I\}_{i, j, k}^{l}
$$

Where $\mathrm{N}$ images and $\mathrm{N}$ normalized weight maps act as alpha masks and each level I of the resulting Laplacian pyramid is computed as a weighted average of the original Laplacian decompositions for level I, with the I-th level of Gaussian pyramid of the weight map serving as the weights. Finally, the pyramid $L\{R\}^{\prime}$ is collapsed to obtain $R[10]$.

In this section a new accuracy evaluation is conducted over the Mertens fusion output thermograms (MEF), taking as comparison reference parameter the TC values. TFDA were combined in 8 ways to evaluate how the generated signal values evolve and its enhancement quality.

Table 3. MEF results TC comparison.

\begin{tabular}{|l|c|c|c|c|}
\hline \multicolumn{1}{|c|}{ MEF } & Sa1 & Sa2 & Sa3 & Sa4 \\
\hline FFTa - LRE_int & -55 & -16 & 50 & 42.85 \\
\hline FFTa - LRE_RGB & 75 & 100 & 60 & 75 \\
\hline nPA - LRE_int & 100 & 100 & 60 & 75 \\
\hline nPA - LRE_RGB & 100 & 100 & 75 & 75 \\
\hline FFTa - LRE_int - LRE_RGB & 75 & 25 & 60 & 33.33 \\
\hline nPA - LRE_int - LRE_RGB & 75 & 60 & 60 & 60 \\
\hline FFTa - nPA - LRE_int & 75 & 25 & 60 & 75 \\
\hline FFTa - nPA - LRE_RGB & 75 & 100 & 60 & 75 \\
\hline
\end{tabular}

As shown above in Table 3, not all combinations lead to a detection accuracy improvement. However, in most cases the accuracy level is increased with respect to its former TC values (in some cases where the former TC level were at $100 \%$, the accuracy level remains equal). Among all fusion combinations, clearly the most robust fusion method is the combination between the nPA and the LRE_RGB. 
In samples Sa1 and Sa2, the highest possible TC value has been obtained and in the nPA / LRE_RGB fusion the value has not been affected. In sample Sa3 the accuracy has been reduced with regard to the TC value obtained in the LRE_RGB. Nevertheless, it is only affected by one false positive and is clearly improved in sample Sa4 case, where the detection accuracy is increased in contrast to its former TC values.
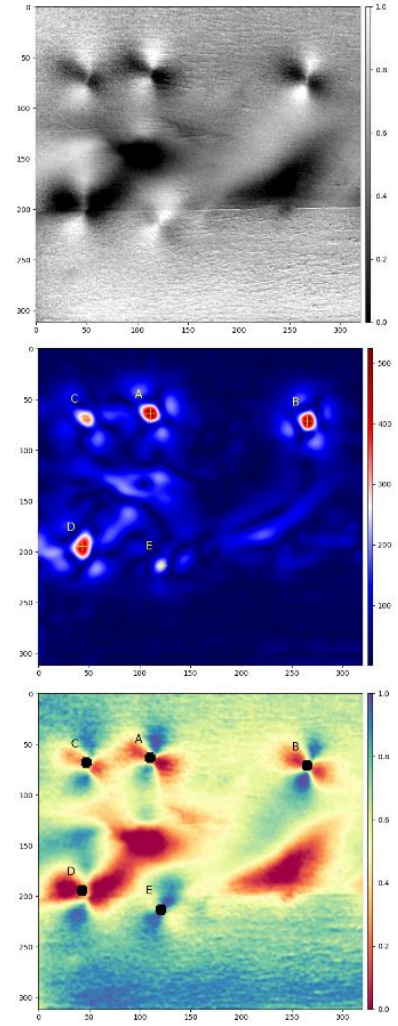

Sa1

\section{nPA - LRE RGB}
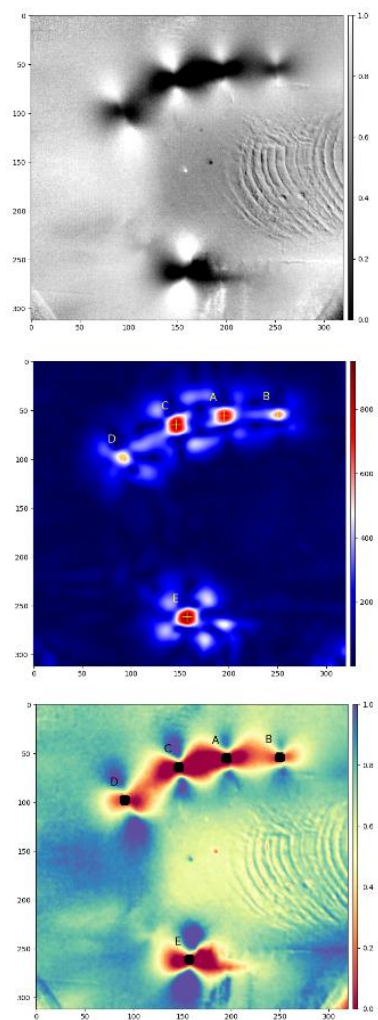

Sa2
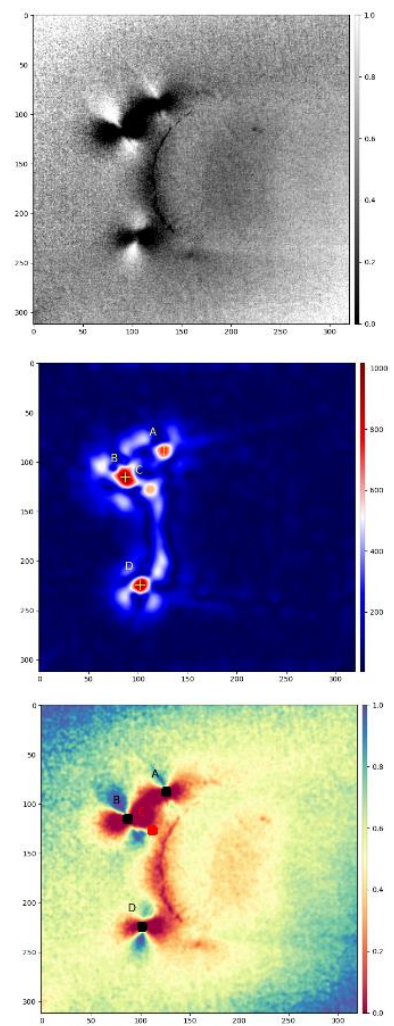

Sa3
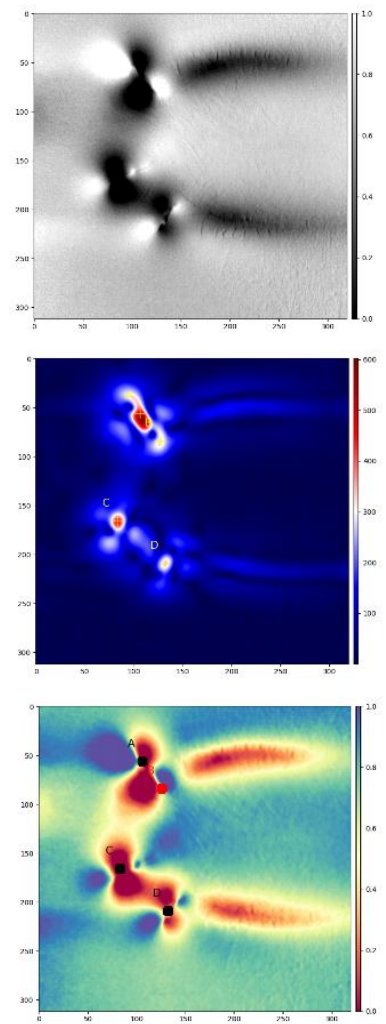

Sa4

Fig. 8. $n P A$ - LRE_RGB fusion thermograms for the selected samples.

\section{Conclusions}

In this study an hybrid processing method has been proposed to improve a previous automated defect detection algorithm. The proposed processing method, based on a time and frequency domain analysis (TFDA), includes a pre-processing part to prepare the thermal signal and reduce the noise and reflexions. The TFDA method generates four thermograms: FFTa phasegram, nPA scaled peaks amplitude, LRE_RGB fusion image from the accumulative sum linear regression parameters and the first interception time instance of the lineal regression evaluation LRE_int (to evaluate the accumulative thermal curve displacement due to defects). These four thermograms by themselves offer very good results in the defect detection, with accuracies near to the $100 \%$ in almost all cases. Thus, a fusion procedure (based on Mertens exposure fusion algorithm) has been evaluated combining the TFDA thermograms. The fusion evaluation results allow the identification of a robust processing algorithm able to ensure and improve the defect detectability. In particular this fusion is the nPA / LRE_RGB Mertens fusion. Finally, the selected hybrid method procedure has been defined as the exposure fusion between the pre-normalized peak amplitude thermogram nPA from the frequency domain analysis and the RGB fusion of LRE parameters LRE_RGB from the time domain analysis. Future work will be focused on (i) the background signal reduction in the processing part and (ii) the pDFT detection algorithm optimization to reduce the false positives and identify with precision the size of the cracks (both length and orientation). 


\section{REFERENCES}

[1] E. G. Colinas, A. Muniategui, P. L. de Uralde, I. Gorosmendi, B. Hériz y X. Sabalza, «A novel Automatic Defect Detection Method for Electron Beam Welded Inconel 718 Components using Inductive Thermography,» de Proceedings of the 2018 International Conference on Quantitative InfraRed Thermography, 2018.

[2] E. Gorostegui-Colinas, R. Hidalgo-Gato, P. L. de Uralde, B. U. Marco y A. Muniategui, «Induction thermography based inspection of EBW and TIG welded Inconel 718 components: steps towards industrialization,» de Thermosense: Thermal Infrared Applications XLII, 2020.

[3] Y. Mei, Y. Liu, C. Liu, C. Li, L. Yu, Q. Guo y H. Li, «Effect of base metal and welding speed on fusion zone microstructure and HAZ hot-cracking of electron-beam welded Inconel 718,» Materials \& Design, vol. 89, p. 964-977, 12016.

[4] S. C. Juang y Y. S. Tarng, «Process parameter selection for optimizing the weld pool geometry in the tungsten inert gas welding of stainless steel,» Journal of Materials Processing Technology, vol. 122, p. 33-37, 32002.

[5] M. S. Węglowski, S. Błacha y A. Phillips, «Electron beam welding - Techniques and trends - Review,» Vacuum, vol. 130, p. 72-92, 82016.

[6] B. L. Luk y A. H. S. Chan, «Human Factors and Ergonomics for Nondestructive Testing,» de Lecture Notes in Electrical Engineering, Springer US, 2008, p. 127-142.

[7] U. Netzelmann, G. Walle, S. Lugin, A. Ehlen, S. Bessert y B. Valeske, «Induction thermography: principle, applications and first steps towards standardization,» de Proceedings of the 2015 Asia International Conference on Quantitative InfraRed Thermography, 2015.

[8] G. Tian, Y. Gao, K. Li, Y. Wang, B. Gao y Y. He, «Eddy Current Pulsed Thermography with Different Excitation Configurations for Metallic Material and Defect Characterization,» Sensors, vol. 16, p. 843, 62016.

[9] S. Sojasi, F. Khodayar, F. López, C. Ibarra-Castanedo, X. Maldague, V. Vavilov y A. Chulkov, «Infrared Testing of CFRP Components: Comparisons of Approaches using the Tanimoto Criterion,» de NDT in Canada 2015 Conference, 2015.

[10] T. Mertens, J. Kautz y F. V. Reeth, «Exposure Fusion: A Simple and Practical Alternative to High Dynamic Range Photography,» Computer Graphics Forum, vol. 28, p. 161-171, 32009.

[11] Yousefi, Ibarra-Castanedo y Maldague, «Infrared Non-Destructive Testing via Semi-Nonnegative Matrix Factorization,» Proceedings, vol. 27, p. 13, 92019.

[12] C. Ibarra-Castanedo y X. Maldague, «Pulsed phase thermography reviewed,» Quantitative InfraRed Thermography Journal, vol. 1, p. 47-70, 62004.

[13] C. I. Castanedo, «Quantitative Subsurface Defect Evaluation by PPT - Depth Retrieval with Phase,» 2005.

[14] J. W. Cooley y J. W. Tukey, «An algorithm for the machine calculation of complex Fourier series, Mathematics of Computation, vol. 19, p. 297-297, 51965.

[15] A. Bekele, «Cooley-Tukey FFT Algorithms,» Advanced Algorithms, 2016. 